\title{
Research on Heat Transfer Characteristics of Impinging Jet on High-Temperature Heating Plate
}

\author{
Zhaohui Wang $^{1, \text { a }}$, Hu Zhang ${ }^{1, b}$ \\ ${ }^{1}$ Beijing Institute of Technology, Beijing 100081, China; \\ awzh_resume@163.com, ${ }^{\mathrm{b}}$ zhanghu@bit.edu.cn,
}

Keywords: impingement jet, numerical simulation, turbulence model, oblique impingement angle, impingement space.

\begin{abstract}
Turbulent impinging slot jets at three different angles and four different impingement spaces were analyzed numaberically using the SIMPLE alogrithm and an RNG-based $\mathrm{k}$ - epsilon turbulence model to investigate the flow field structure and heat transfer characteristics. Under a certain condition, the heat board of a device is cooled by impinging jet, designed three Different jet angles, including $35^{\circ}, 45^{\circ}$ and $55^{\circ}$, The results show that as the angle becomes large, the air convection in the low temperature region becomes stronger, and the heat exchange rate is larger in the high air domain. Namely, when the oblique impingement angle increases, the convective heat transfer in the air field at both sides of the low temperature zone and the high temperature zone is more severe, which is not conducive to the cooling of the core area. Four different partition schemes were designed to form different impinging jet space, the results show that in a certain range, the larger the jet space, the air field will have a larger vortex, which will reduce the cooling efficiency.
\end{abstract}

\section{Introduction}

Impinging jet is the impact flow of the jet on the solid wall or liquid surface, Because the fluid directly impact the surface of heating or cooling, flow boundary layer on the surface of the impacted surface is thin and the short process, the gas or liquid in the role of pressure difference, with a round or narrow vertical nozzle (or a certain angle) is injected into the cooling or heating on the surface, so that the impact of the regional heat transfer effect is strong, that is why impinging jet has been widely used in many engineering fields ${ }^{[1-3]}$. The heat transfer process of the impinging jet involves many factors, including the temperature of the impacted surface, the physical parameters of the medium, the impact mode, the geometrical parameters and the arrangement of the nozzle. Therefore, this process is very complex.

In this paper, considering the above factors, in a device for heating plate received high temperature shock, the impact of different factors on the impact cooling is simulated under certain conditions, and the final cooling scheme is determined by the software analysis, and the heat transfer law of the impinging jet is studied.

\section{Cooling Device Structure Design}

The design of a heat insulation structure, in order to reduce or block the high temperature heating plate heat convection and radiation to other structures in the cabin, centralized cooling of nuclear heating plate, so as to improve the cooling efficiency, and put forward the insulation board in the cabin of air cooling scheme. The specific structure is shown in Figure 1, including insulation layer, heated plate, heat shield, air inlet and outlet, where $\theta$ is the jet angle, namely the angle between the vertical direction and speed of air. The impinging jet space is surrounded by heating plate, heat insulating board and heat insulating layer. 


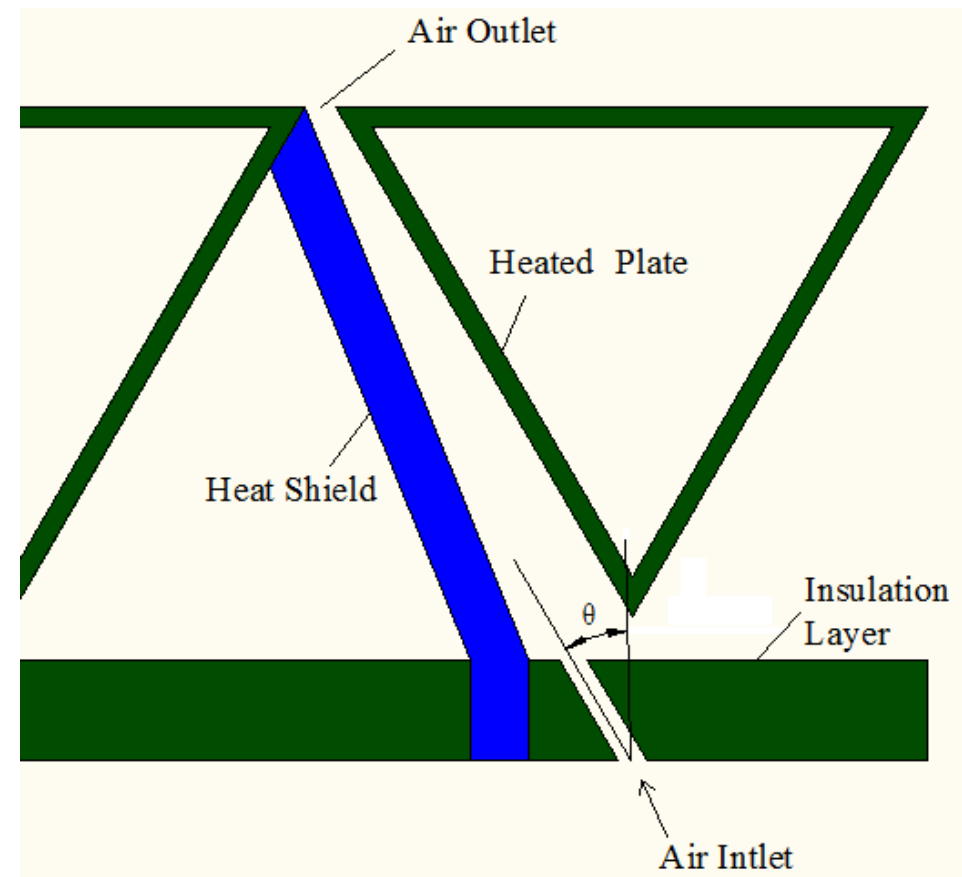

Fig. 1 Geometric Structure

In order to examine the influence of different factors on the effect of jet impingement, set up different jet blowing angle and different cabin partition structure, namely the different angle and different jet space on the influence of turbulence slit impact jet flow and heat transfer performance, CFD ICEM is used to mesh the model, and then the FLUENT is used to simulate the fluid dynamics, to obtain the temperature distribution and the temperature change curve of the cooling device.

\section{Mathematical Model of the Impingement Jet}

Consider the impact jet flow in the space of a semi enclosed (with a insulating board) as shown in figure 1, and the medium is air, imported jet Reynolds number $\operatorname{Re}=10000(\operatorname{Re}=\rho v d / \mu,(v, \rho, \mu$ are fluid velocity, density and air motion viscosity coefficient). Under the condition of unsteady incompressible hypothesis, the flow and heat transfer phenomena in the turbulent impinging jet flow field can be described by the Reynolds averaged Naiver-Stokes equation and the energy equation as follows:

$$
\begin{gathered}
\frac{\partial u_{i}}{\partial x_{i}}=0 \\
u_{j} \frac{\partial u_{i}}{\partial x_{j}}=-\frac{1}{\rho} \frac{\partial p}{\partial x_{i}}+\frac{\partial}{\partial x_{j}}\left[\left(v+v_{t}\right)\left(\frac{\partial u_{i}}{\partial x_{j}}+\frac{\partial u_{j}}{\partial x_{i}}\right)\right] \\
u_{j} \frac{\partial T}{\partial x_{j}}=\frac{\partial}{\partial x_{j}}\left[\left(\frac{v}{P r}+\frac{v_{t}}{P r_{t}}\right) \frac{\partial T}{\partial x_{j}}\right]
\end{gathered}
$$

$x_{i}$ and $x_{j}$ in the formula ( $\left.\mathrm{i}, \mathrm{j}=1,2,3\right)$ for Cartesian coordinates, $\rho$ is the fluid (air) density, $u_{i}$ and $u_{j}(\mathrm{i}, \mathrm{j}=1,2,3)$ for the average speed, $\mathrm{p}$ for the average pressure, $v$ and $v_{t}$ are molecular viscosity and turbulent viscosity. Pr in the energy equation is the Pradtl number; $P r_{t}$ is the turbulent Pradtl number ${ }^{[7]}$.

Based on the turbulence $\mathrm{k}$ - epsilon model for both the fluid flow in thermal transfer control equation including continuity equation, motion equation and energy equation. The form of the partial differential control equations, with appropriate initial and boundary conditions, and the discrete numerical solutions of velocity fields, pressure fields and temperature fields can be solved by using the finite element method ${ }^{[4-5]}$. The RNG k-epsilon model is adopted in this paper ${ }^{[6]}$, which is similar to standard $\mathrm{k}$ - epsilon model. However, the standard k-epsilon model is a model of high Reynolds number, the RNG k-epsilon theory provides a thinking of low Reynolds number flow viscosity analytic formula, makes RNG k-epsilon more applicable to this paper than the standard k-epsilon model. 
Equation of RNG k-epsilon model:

$$
\begin{gathered}
\frac{D(\rho \mathrm{k})}{D \mathrm{t}}=\frac{\partial}{\partial x_{j}}\left[\left(\mu+\frac{\mu_{t}}{\sigma_{k}}\right) \frac{\partial_{k}}{\partial x_{j}}\right]+G_{k}-\rho \epsilon \\
\frac{D(\rho \epsilon)}{D \mathrm{t}}=\frac{\partial}{\partial x_{j}}\left[\left(\mu+\frac{\mu_{t}}{\sigma_{\epsilon}}\right) \frac{\partial_{\epsilon}}{\partial x_{j}}\right]+C_{1 \epsilon} \frac{\epsilon}{k} G_{k}-C_{2 \epsilon} \rho \frac{\varepsilon^{2}}{k}
\end{gathered}
$$

The turbulent viscosity coefficient $\mu_{t}$ in the equation is defined as:

$$
\mu_{t}=\rho C_{\mu} \frac{k^{2}}{\epsilon}
$$

$G_{k}$ is the generation term of the turbulent kinetic energy due to the velocity gradient:

$$
G_{k}=-\rho u_{i} u_{j} \frac{\partial u_{j}}{\partial x_{i}}
$$

The turbulence coefficient in the equation are:

$$
C_{\mu}=0.085, \sigma_{k}=0.7179, \quad \sigma_{\epsilon}=0.7179, \quad C_{1 \epsilon}=1.44, \quad C_{2 \epsilon}=1.92
$$

\section{Boundary Conditions Setting}

The width of the air inlet is $10 \mathrm{~mm}$, and the wind speed is $10 \mathrm{~m} / \mathrm{s}$;

The width of the outlet is $10 \mathrm{~mm}$, and the flow velocity is 0 , which is close to the wall surface;

The initial temperature of the heated plate is $1000 \mathrm{~K}, \mathrm{~g}_{\mathrm{y}}=9.81 \mathrm{~kg} / \mathrm{m}^{3}$;

The type of cooling gas inlet is set to mass-flow-inlet, $Q=1.3983424 \mathrm{~kg} / \mathrm{s}$;

The export type is set to pressure-outlet, Atmospheric Pressure $\mathrm{P}=1.013 \times 10^{5} \mathrm{P}_{\mathrm{a}}$.

\section{Analysis and Results}

\section{Analysis of Different Blowing Angles}

In order to screen out the appropriate wind blowing angle, set up a total of three angles, respectively $35^{\circ}, 45^{\circ}$ and $55^{\circ}$. The inlet wind speed is $10 \mathrm{~m} / \mathrm{s}$, the cooling time is $60 \mathrm{~s}$, angle respectively temperature images of $35^{\circ}, 45^{\circ}$ and $55^{\circ}$ is shown in figure 2 to 4 , as can be seen, when the inlet angle is $35^{\circ}$, the temperature of the left side of the air field is low for $30{ }^{\circ} \mathrm{C}$; And the air inlet angle is $45^{\circ}$ and $55^{\circ}$, the left side of the air field gas temperature is higher, respectively $75{ }^{\circ} \mathrm{C}$ and $100{ }^{\circ} \mathrm{C}$. This is because the larger the angle, the stronger the left air field convection, the amount of heat exchange larger with the hot air domain. When the air inlet angle is increased, the convective heat transfer in the air space between the left and the right sides is quite severe. In conclusion, when the wind speed is $10 \mathrm{~m} / \mathrm{s}$, the angle is $35^{\circ}$, the cooling effect is better.

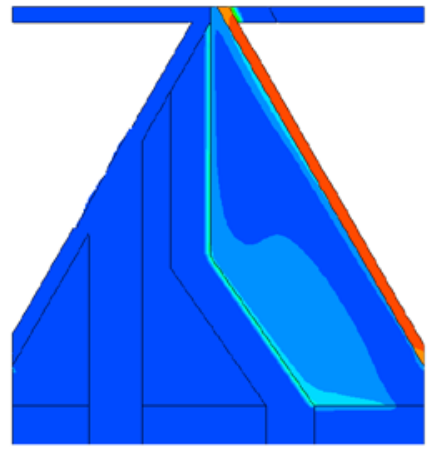

Time $=15 \mathrm{~s}$

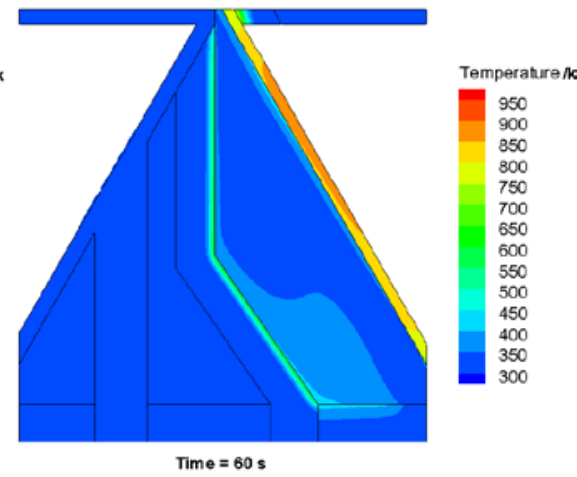

Time $=60 \mathrm{~s}$

Figure 2. Angle of $35^{\circ}$, cooling 15 s and 60s corresponding temperature cloud 


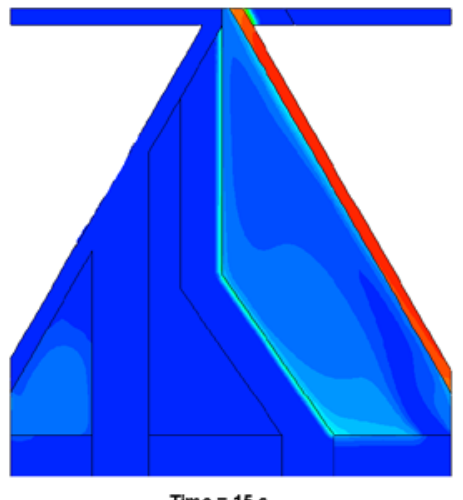

Time $=15 \mathrm{~s}$

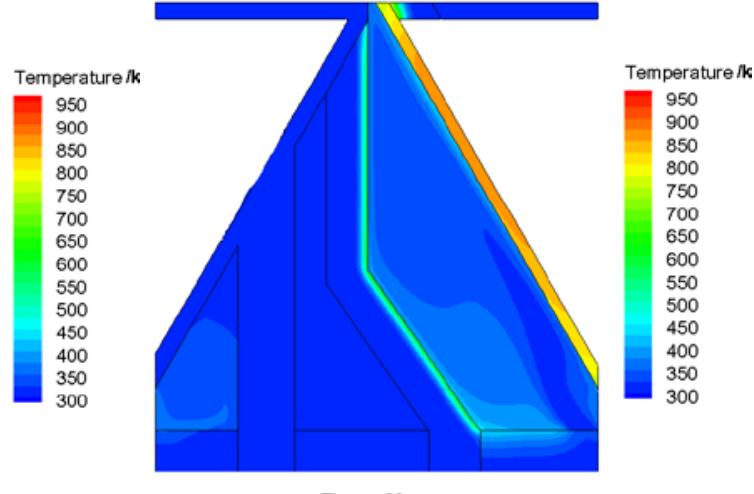

Figure 3. Angle of $45^{\circ}$, cooling 15 s and 60 s corresponding temperature cloud

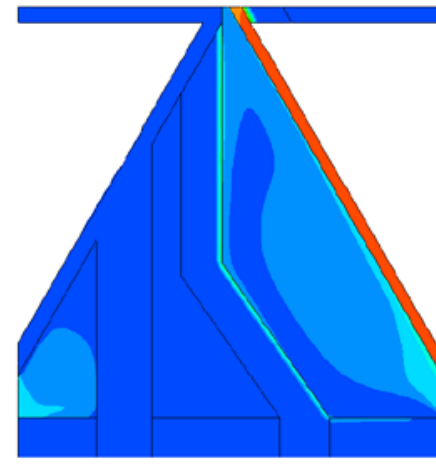

Time=15 s

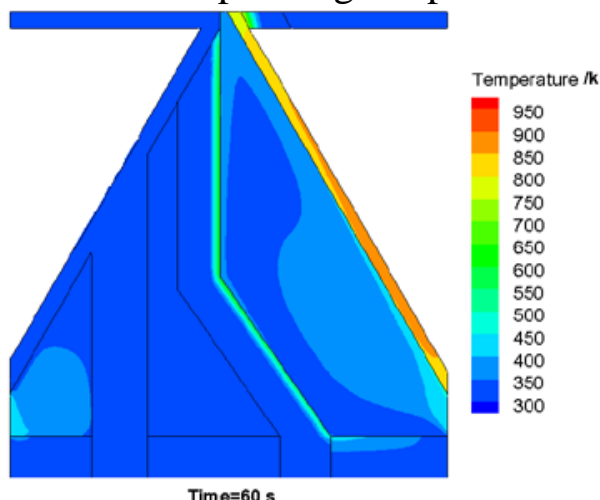

Time $=60 \mathrm{~s}$

Figure 4. Angle of $55^{\circ}$, cooling 15s and 60s corresponding temperature cloud

\section{Analysis of Different Partition Structures}

Due to the change of heat shield position and shape will affect the cooling effect of the heating plate and the exit gas temperature, so it is necessary to optimize the location and shape of insulation board, and then form different impingement jet space, so as to select the best cooling scheme.

Comparative analysis of four kinds of partition structure, and the specific structure shown in Figure 5 , four kinds of structure of the jet space can be expressed by volume $\mathrm{v}$, namely impingement space of structure No. 1 is v, No. 2 is $5 \mathrm{v}$, No. 3 is $3 \mathrm{v}$, the volume of No. 4 is similar to No. 3, but the insulation board have bent so that the jet space is relatively smoother. The initial temperature distribution of the heating plate and other boundary conditions are the same as above, the size of the inlet and outlet is $10 \mathrm{~mm}$, the wind speed is $10 \mathrm{~m} / \mathrm{s}$, the inlet angle is $35^{\circ}$.

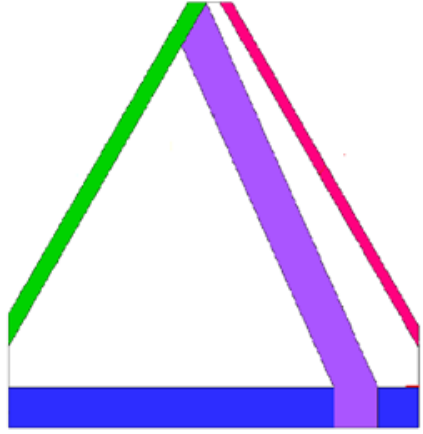

(a) No. 1

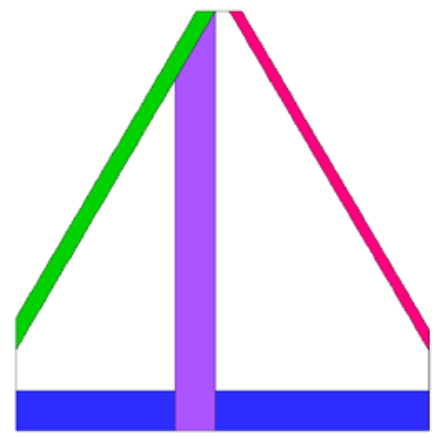

(b) No. 2 


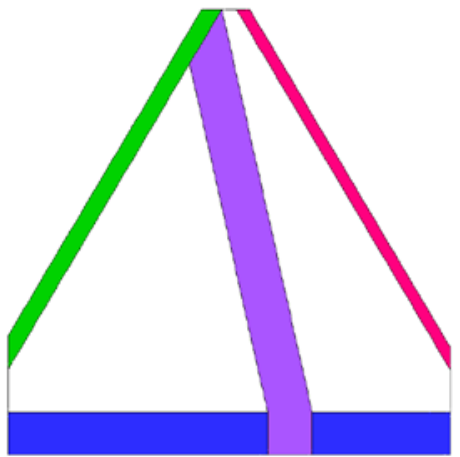

(c) No. 3

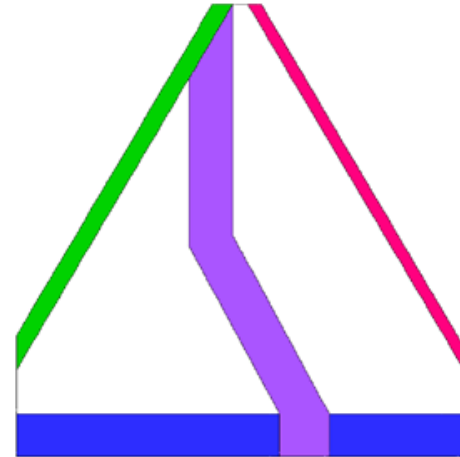

(d) No. 4

Fig. 5 Schematic diagram of different partition structure

(1) Temperature contour and velocity vector diagram after cooling $15 \mathrm{~s}$

Figure $6 \sim 9$ for the 4 kinds of partition structure corresponding to the temperature and speed vector map, the map can be seen from the figure the larger the space, the greater the vortex.

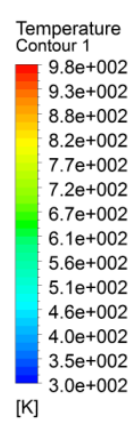

(a) Temperature contour

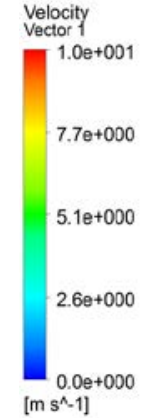

Figure 6 Structure No. 1

(b) Velocity vector

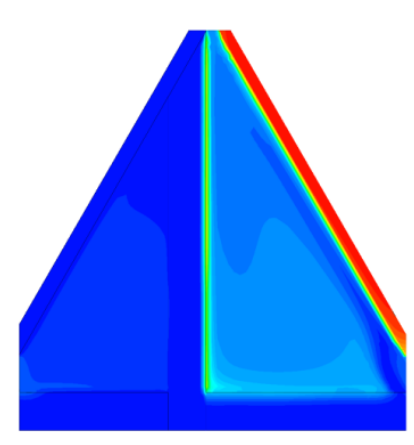

a) Temperature contour

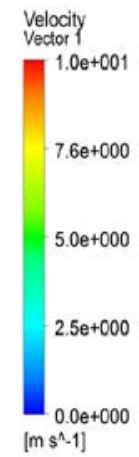

[m $\mathrm{s}^{n-1]}$

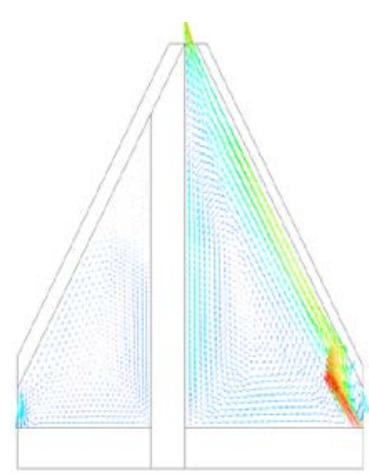

(b) Velocity vector

Figure 7 Structure No. 2
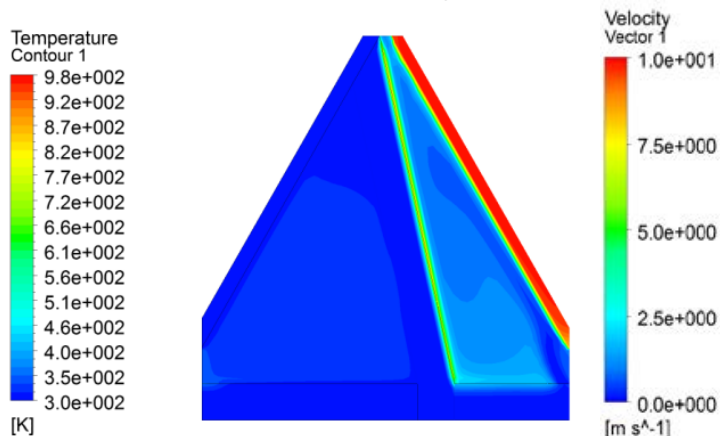

(a) Temperature contour

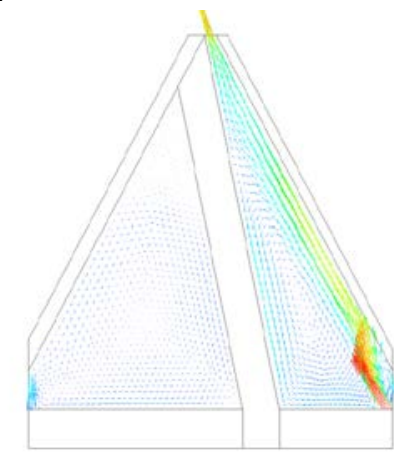

(b) Velocity vector

Figure 8 Structure No. 3 


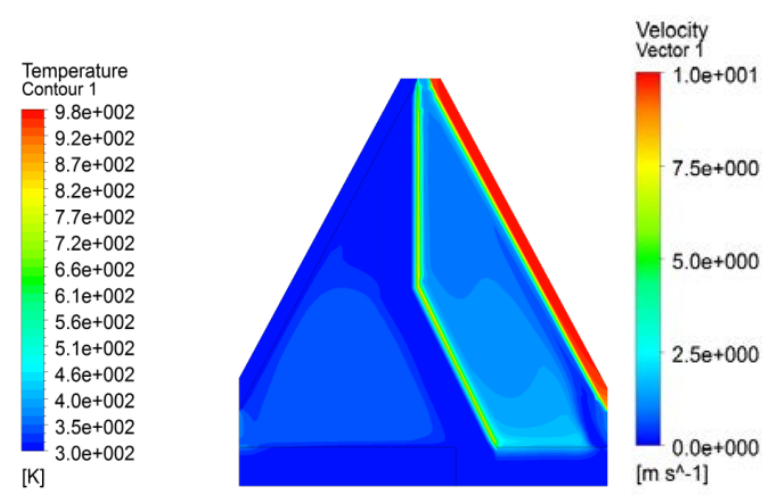

(a) Temperature contour

Figure 9 Structure No. 4

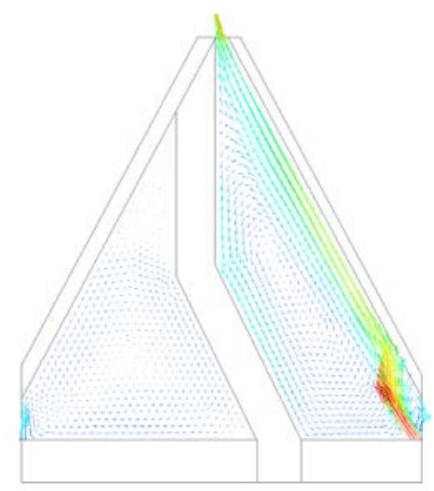

(b) Velocity vector

(2) Comparative analysis of average temperature and maximum temperature contrast curves of four kinds of partition structures

As can be seen from Figure 10, the temperature difference of the average temperature and maximum temperature of the air domain 1 and the heated plate of the four kinds of partitioning structures is little. From the exit gas temperature curve, we can see that the No. 1 structure and No. 4 structure is better, the outlet gas average temperature of No.1 structure is $133.2{ }^{\circ} \mathrm{C}$ and the maximum temperature is $145.1{ }^{\circ} \mathrm{C}$; the average outlet gas temperature of No.4 structure is $126.9{ }^{\circ} \mathrm{C}$, the maximum temperature is $163.5{ }^{\circ} \mathrm{C}$; So comprehensive consideration, No. 1 structure namely the smaller impingement jet space the effect is better.

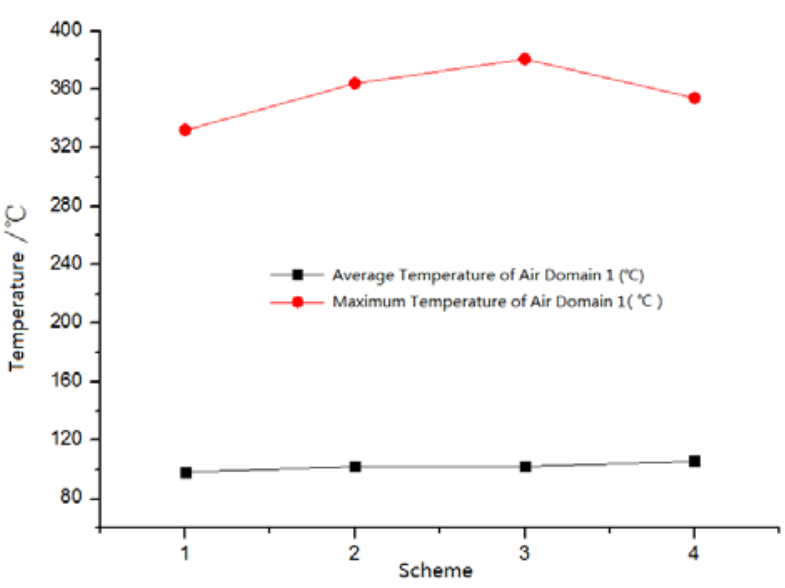

(a) Temperature of air domain 1

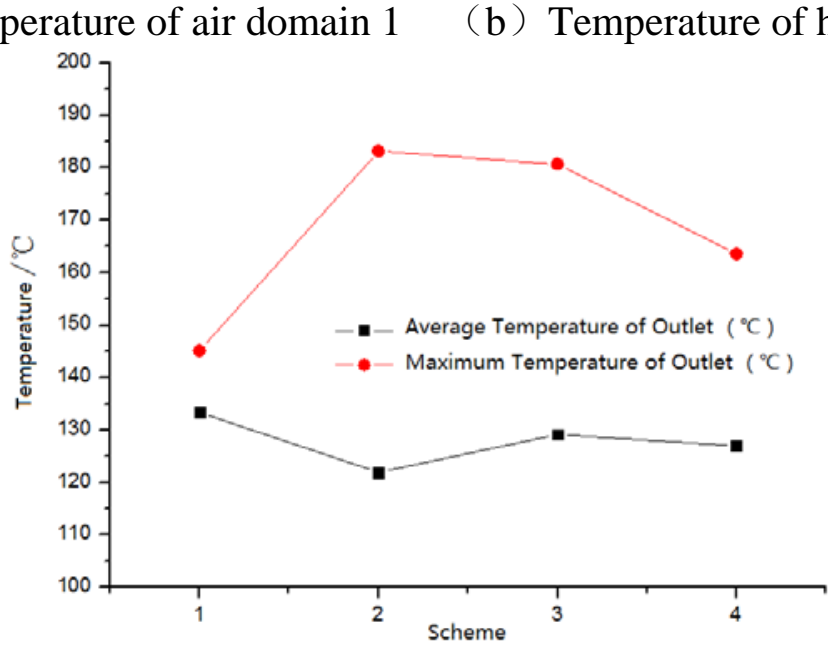

(c) Temperature of outlet gas

Fig.10 Comparison curves of average temperature and minimum temperature of four schemes (3) The results of the calculation of the structure No. 1 for cooling 20min

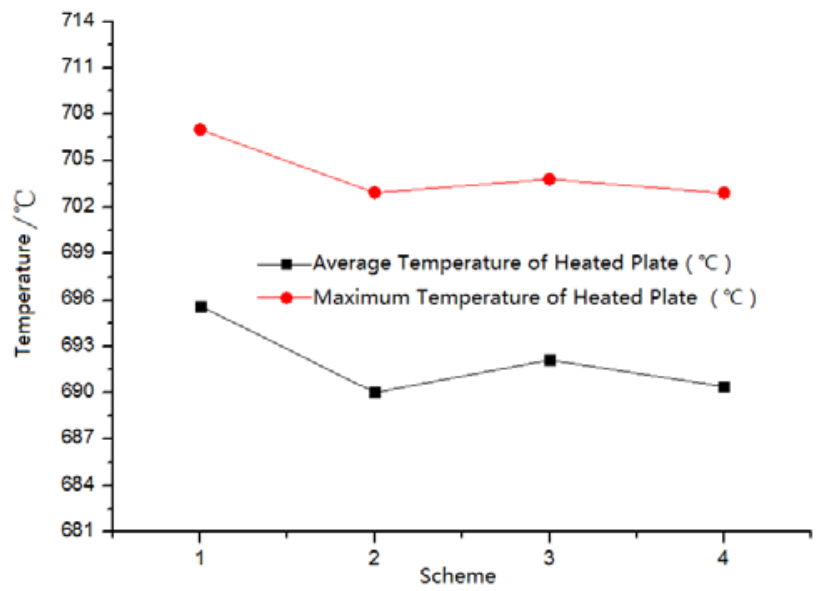

(b) Temperature of heated plate 
Figure 11 shows the export temperature change curve, can be seen from the figure, the outlet temperature in the cooling $24 \mathrm{~min}$, the average temperature is $320 \mathrm{~K}$, the maximum temperature is $400 \mathrm{~K}$. Figure 12 shows the temperature variation curve of the heating plate, can be seen from the figure, after 24 min of cooling, heating plate temperature changing with time began to slow, the average temperature of high temperature heating plate is $364.49 \mathrm{~K}$, the maximum temperature is $396.75 \mathrm{~K}$.
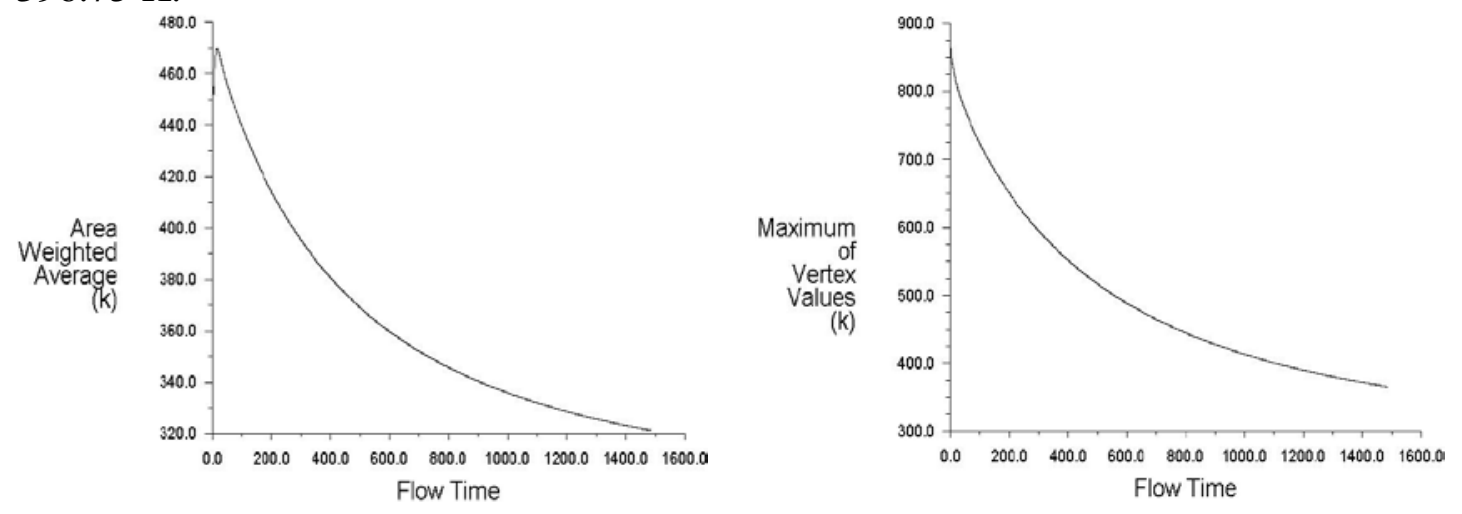

temperature variation curve

(b) Maximum temperature change curve

Fig. 11 Curve of outlet temperature over time

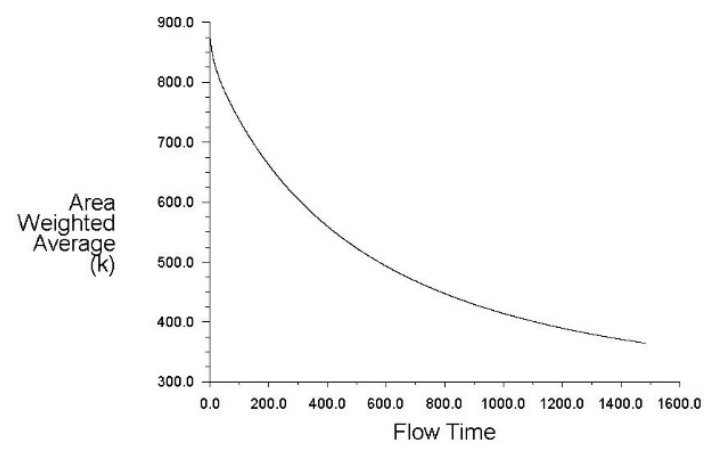

temperature variation curve

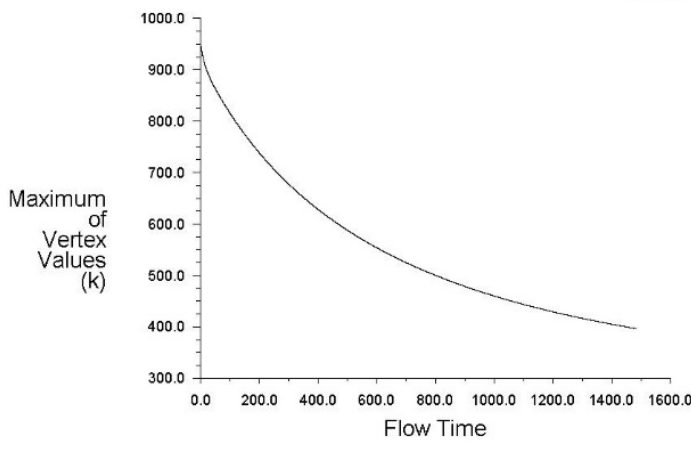

(b) Maximum temperature change curve (a) Average

(a) Average

Fig. 12 Curve of heated plate temperature over time

\section{Summary}

In this paper, numerical simulation is used to study the influence of different inclination angles and different impingement spaces on the heat transfer performance of turbulent impinging jets in a certain range. The results show that impingement angle and impinging jet space have significant influence on the flow field structure and heat transfer performance:

(1) With the decrease of the jet angle, the mean Nu value of the impinging surface increases with the increase of the angle between the jet and the heated plate, resulting in an increase in the average heat transfer rate of the jet, thus improving the cooling efficiency.

(2) Different impingement jet space formed by the different compartment partition scheme, the larger the impingement jet space in a certain range, it will lead to a larger vortex in the air field, thereby reducing the cooling efficiency.

\section{References}

[1] GENG Tie, Li-De qun, ZHOU hua-min, SHAO Yu-jie, WANG Wei-jun, Development of the studies about heat transfer with impinging jets, Machinery Dedign \& Manufacture, 2006, (6): 154-156.

[2] ZHANG Jing-zhou, LI Yong-kang, TAN Xiao-ming, LI Li-guo, Numerical Computation and Experimental Investigation on Local Convective Heat Transfer Characteristics for Jet Array Impingement, ACTA AERONAUTICA ET ASTRONAUTICA SINICA, 2004, 25(4): 339- 342. 
[3] TAN Lei, ZHANG Jing -zhou, TAN Xiao-ming, Numerical Investigation of Jet Impingement Cooling with Non-Uniform Crossflow, Journal of Aerospace Power, 2006, 21(3): 528-532.

[4] CHEN Qing-guang, Wu Yu-lin, ZHANG Yong-jian, Numerical study on flow and heat transfer of turbulent impinging jets in a rectangular duct, Journal of Engineering for Thermal Energy and Power, 2005, (5): 474-477.

[5] Niu Jue, Wen Zhi, Wang Jun-sheng, Numerical simulation of flow and heat transfer of a round nozzle turbulent impinging jet flow, Energy for metallurgical industry, 2007, 26(1), 16-20.

[6] CHEN Qing-guang, XU Zhong, ZHANG Yong-jian, Numerical study of turbulent impinging jet flow and heat transfer, Advances in Mechanics, 2002, 32(1), 92-108.

[7] CHEN Qing-guang, WU Yu-lin, LIU Shu-hong, ZHANG Yong-jian, Effect of oblique angle on flow and heat transfer characteristics of a turbulent impinging slot jet, Journal of Tsinghua University(Science and Technology), 2005, 45(8), 1114-111. 